PROCEEDINGS OF THE

AMERICAN MATHEMATICAL SOCIETY

Volume 137, Number 8, August 2009, Pages 2733-2740

S 0002-9939(09)09789-5

Article electronically published on April 3, 2009

\title{
THE SIZE OF ISOPERIMETRIC SURFACES IN 3-MANIFOLDS AND A RIGIDITY RESULT FOR THE UPPER HEMISPHERE
}

\author{
MICHAEL EICHMAIR
}

(Communicated by Richard A. Wentworth)

\begin{abstract}
We characterize the standard $\mathbb{S}^{3}$ as the closed Ricci-positive 3manifold with scalar curvature at least 6 having isoperimetric surfaces of largest area: $4 \pi$. As a corollary we answer in the affirmative an interesting special case of a conjecture of M. Min-Oo's on the scalar curvature rigidity of the upper hemisphere.
\end{abstract}

\section{INTRODUCTION}

The following rigidity result for the unit ball of $\left(\mathbb{R}^{3}, \delta\right)$ is a well-known consequence of the positive mass theorem (see [16], 19, [1], 17], and 8]):

Theorem. Let $\left(M^{3}, g\right)$ be a compact orientable Riemannian 3-manifold with nonnegative scalar curvature and boundary isometric to round $\mathbb{S}^{2}$ with mean curvature equal to 2 . Then $\left(M^{3}, g\right)$ is isometric to the unit ball of $\left(\mathbb{R}^{3}, \delta\right)$.

The theorem asserts that there are no compact deformations of the Euclidean metric within the class of non-negative scalar curvature metrics. The analogous rigidity statement for hyperbolic space was proven in [12, [18, [5], [1 by establishing appropriate versions of the positive mass theorem in this context. In [13] M. Min-Oo raised the following question:

Conjecture (Min-Oo). Let $\left(M^{n}, g\right)$ be an n-dimensional compact orientable Riemannian manifold with scalar curvature $\mathrm{R} \geq n(n-1)$ and totally geodesic boundary isometric to round $\mathbb{S}^{n-1}$. Then $\left(M^{n}, g\right)$ is isometric to the round hemisphere $\mathbb{S}_{+}^{n}$.

We refer the reader to [13, 8], and [9] for more background and context for Min-Oo's conjecture. Recall that the standard metrics on $\mathbb{R}^{n}, \mathbb{H}^{n}$, and $\mathbb{S}^{n}$ are all static (the linearization of the scalar curvature map about the standard metrics on these spaces has non-trivial cokernel). By the work of J. Corvino (Theorem 4 in [6]), one can always locally deform the scalar curvature in any direction if the underlying metric is not static:

Theorem (Corvino). Let $\Omega$ be a smooth domain compactly contained in a Riemannian manifold $\left(M^{n}, g_{0}\right)$. Suppose that the linearization $L_{g_{0}}$ of the scalar curvature map $\mathrm{R}: \mathcal{C}^{\infty}(\Omega) \rightarrow \mathcal{C}^{\infty}(\Omega)$ at $g_{0}$ has an injective formal $\mathcal{L}^{2}$-adjoint $L_{g_{0}}^{*}$, where we consider $L_{g_{0}}: H_{l o c}^{2}(\Omega) \rightarrow \mathcal{L}_{\text {loc }}^{2}(\Omega)$. Then for every smooth function $S$ on $\Omega$

Received by the editors December 3, 2007, and, in revised form, September 17, 2008.

2000 Mathematics Subject Classification. Primary 53C20.

(C)2009 American Mathematical Society 
sufficiently close to the scalar curvature $\mathrm{R}\left(g_{0}\right)$ and equal to $\mathrm{R}\left(g_{0}\right)$ near $\partial \Omega$, there exists a smooth metric $g$ on $\Omega$ such that $\mathrm{R}(g)=S$ and so that $g \equiv g_{0}$ outside $\Omega$.

As was noted in [8], by Corvino's theorem, staticity appears as an obstruction to finding potential counterexamples to Min-Oo's conjecture near the round metric on $\mathbb{S}_{+}^{n}$. However, in $[8, \mathrm{~F}$. Hang and X. Wang show that if one moves from the hemisphere $\mathbb{S}_{+}^{n}$ to a larger geodesic ball of $\mathbb{S}^{n}$, there even are metrics conformally related to the round metric with scalar curvature $\geq n(n-1)$ and standard boundary geometry:

Theorem (Hang and Wang). For any $r \in\left(\frac{\pi}{2}, \pi\right)$ there is a smooth metric $g=$ $e^{2 \phi} g_{\mathbb{S}^{3}}$ on $\mathbb{S}^{3}$ such that (a) $\mathrm{R}_{g} \geq 6$, (b) $\phi$ is not identically 0 , and (c) $\operatorname{supp}(\phi) \subset$ $B(N, r)$, where $N$ is a fixed point in $\mathbb{S}^{3}$.

By contrast, in the same paper the authors establish Min-Oo's conjecture among conformal deformations:

Theorem (Hang and Wang). Let $g=e^{2 \varphi} g_{\mathbb{S}^{n}}$ be a $\mathcal{C}^{2}$-metric on the upper hemisphere $\mathbb{S}_{+}^{n}$ satisfying the assumptions $(a) \mathrm{R}_{g} \geq n(n-1)$ and (b) the boundary is totally geodesic and isometric to standard $\mathbb{S}^{n-1}$. Then $g$ is isometric to $g_{\mathbb{S}^{n}}$.

In a recent paper [9], F. Hang and X. Wang use Raleigh's Bôchner-type formula on manifolds with boundary to show that Min-Oo's conjecture holds true in all dimensions if one adds $\operatorname{Ric}_{M} \geq(n-1) g$ to the hypotheses.

The main result of this work answers Min-Oo's conjecture in the affirmative in the case where $n=3, \operatorname{Ric}_{M}>0$, and the boundary is an isoperimetric surface (Theorem 2):

Theorem. Let $\left(M^{3}, g\right)$ be a compact orientable Riemannian 3-manifold with scalar curvature $\mathrm{R}_{M} \geq 6$, Ricci curvature $\operatorname{Ric}_{M}>0$ and totally geodesic boundary $\partial M^{3}$. If area $\left(\partial M^{3}\right) \geq 4 \pi$ and $\partial M^{3}$ is an isoperimetric surface for the doubled manifold $\left(\hat{M}^{3}, \hat{g}\right)$, then $\left(M^{3}, g\right)$ is isometric to the upper hemisphere $\mathbb{S}_{+}^{3}$.

We point out that it is not necessary here to assume that $\partial M^{3}$ is round. In fact, the author is not aware of counterexamples to Min-Oo's conjecture when the original condition on the inner geometry of $\partial M$ is weakened to a lower bound on its area, as above.

Our main contribution here, which quickly leads to a proof of the above theorem, is to characterize round $\mathbb{S}^{3}$ as the unique Ricci-positive 3-manifold with scalar curvature at least 6 that admits isoperimetric surfaces of largest area (Theorem 11):

Theorem. Let $\left(M^{3}, g\right)$ be a closed Riemannian manifold with $\mathrm{R}_{M} \geq 6$ and $\operatorname{Ric}_{M}>$ 0 . Then all isoperimetric surfaces of $M^{3}$ have area strictly less than $4 \pi$ unless $\left(M^{3}, g\right)$ is isometric to $\mathbb{S}^{3}$.

There are two steps in the proof of this theorem. The crucial first ingredient is the monotonicity of a certain isoperimetric mass that $\mathrm{H}$. Bray discovered in his thesis [3]. H. Bray's arguments imply directly that the isoperimetric profile of $\left(M^{3}, g\right)$ coincides with that of round $\mathbb{S}^{3}$. We recall that the isoperimetric surfaces of $\mathbb{S}^{3}$ are the geodesic balls (this follows from a symmetrization argument; see for example [14]). Second, to show that $\left(M^{3}, g\right)$ is in fact round itself, we employ a delicate comparison with the isoperimetric ratio of small geodesic balls in $M^{3}$, using the Taylor expansion in 7 for their volume. Note that the theorem shows how the 
global "low order" information about the manifold $\left(M^{3}, g\right)$ that is contained in the isoperimetric assumption leads to complete rigidity of the local geometry.

H. Bray's results in [3] have not been published. In the next section, we will summarize the required portion of his method to explain how it applies in this paper.

Note that the upper bound of $4 \pi$ on the size of isoperimetric surfaces in the preceding theorem is already contained in the work of D. Christodoulou and S.-T. Yau 4 and comes out of a Hersch-type choice of test functions in the stability inequality:

Theorem (Christodoulou and Yau). Let $\left(M^{3}, g\right)$ be a Riemannian 3-manifold and let $\Sigma^{2} \subset M^{3}$ be an immersed closed (weakly) stable constant mean curvature surface of genus 0 . Then

$$
16 \pi \geq \int_{\Sigma} \mathrm{H}_{\Sigma}^{2}+\frac{2}{3} \int_{\Sigma} \mathrm{R}_{M}
$$

where $\mathrm{R}_{M}$ is the scalar curvature of $M^{3}$.

We point out that in general relativity very successful notions of mass and quasilocal mass based on the isoperimetric defect (from Schwarzschild) of surfaces have been introduced by G. Huisken. In conjunction with monotonicity formulae along mean and inverse mean curvature flow, he has proven positive mass and Penrosetype theorems for these notions of mass. His work has not been published at this point, but see the Oberwolfach report [10].

\section{Proofs}

We review some standard results regarding the isoperimetric profile function of a closed Riemannian manifold, starting with its definition. We refer to the excellent survey article [14 and the paper [2] for the history and basic properties of the isoperimetric profile, as well as for further references on this topic.

Definition 1 (2]). Given a closed Riemannian 3-manifold $\left(M^{3}, g\right)$, define its isoperimetric profile function $I:\left[0, \operatorname{vol}\left(M^{3}\right)\right] \rightarrow \mathbb{R}$ by

$$
I(V)=\inf \left\{\operatorname{area}(\partial \Omega): \Omega \subseteq M^{3} \text { region with } \operatorname{vol}(\Omega)=V\right\} .
$$

It is a classical result in geometric measure theory that the infimum in the definition of the isoperimetric profile is achieved by "isoperimetric regions" $\Omega \subset M^{3}$ with smooth embedded boundaries $\Sigma^{2}=\partial \Omega$. Surfaces $\Sigma^{2} \subset M^{3}$ arising in this way are called isoperimetric surfaces. A standard first variation argument yields that isoperimetric surfaces have constant mean curvature (the same constant for all connected components). The definition implies that $I(V)$ is symmetric with respect to $\frac{1}{2} \operatorname{vol}\left(M^{3}\right)$.

The following basic regularity of the isoperimetric profile was established in [2]:

Lemma 1 (2]). Given $\left(M^{3}, g\right)$ closed and $V \in\left(0, \operatorname{vol}\left(M^{3}\right)\right)$, let $\Omega \subset M^{3}$ be an isoperimetric region with $\operatorname{vol}(\Omega)=V$ and denote $\partial \Omega=\Sigma^{2}$. Write $\mathrm{A}_{\Sigma}, \mathrm{H}_{\Sigma}$ for the second fundamental form and (constant) mean curvature of $\Sigma^{2}$ computed with respect to its outward normal $\vec{\nu}$. The isoperimetric profile has the following regularity:

a) $I$ has left and right derivatives at $V$, and $I^{\prime+}(V) \leq \mathrm{H}_{\Sigma} \leq I^{\prime-}(V)$.

b) $I^{\prime \prime}(V) I(V)^{2}+\int_{\Sigma}\left(\operatorname{Ric}_{M}(\vec{\nu}, \vec{\nu})+\left|\mathrm{A}_{\Sigma}\right|^{2}\right) \leq 0$ holds in the sense of comparison functions. 
The lemma asserts that for every $V \in\left(0, \operatorname{vol}\left(M^{3}\right)\right)$ there exists a smooth function $I_{V}$ defined in a neighbourhood of $V$ such that $I_{V}(V)=I(V), I_{V} \geq I$, and $I_{V}^{\prime \prime}(V) I_{V}^{2}(V)+\int_{\Sigma}\left(\operatorname{Ric}_{M}(\vec{\nu}, \vec{\nu})+\left|\mathrm{A}_{\Sigma}\right|^{2}\right) \leq 0$. A well-known and immediate consequence of part b) is that the isoperimetric profile is concave when $\left(M^{3}, g\right)$ has non-negative Ricci curvature.

In his thesis, H. Bray proved a scalar curvature based volume comparison theorem for 3-manifolds. More precisely, he showed that if a closed 3-manifold $\left(M^{3}, g\right)$ has scalar curvature bounded below by 6 and Ricci curvature bounded below by $\varepsilon(2 g)$ for some $\varepsilon \in(0,1)$, then its volume is bounded above by the volume of the round unit sphere $\mathbb{S}^{3}$ times a constant $\alpha(\varepsilon)$, where $\alpha(\varepsilon)=1$ for large enough $\varepsilon \in(0,1)$. The techniques from [3], which are crucial in our proof of Theorem [1, have not been published. For convenient reference and in order to explain how the arguments from [3] apply directly in our context, we summarize several statements and proofs from H. Bray's thesis below:

Definition $2\left([3)\right.$. Let $\left(M^{3}, g\right)$ be a closed Riemannian 3-manifold with scalar curvature $\mathrm{R}_{M} \geq 6$. The adapted Hawking $m_{H}:\left(0, \operatorname{vol}\left(M^{3}\right)\right) \rightarrow \mathbb{R}$ is defined in terms of the isoperimetric profile by

$$
m_{H}(V)=\sqrt{I(V)}\left(16 \pi-4 I(V)-I(V) I^{\prime+}(V)^{2}\right) .
$$

It was proven in $[3]$ that isoperimetric surfaces are connected if the ambient manifold has positive Ricci curvature. In conjunction with the Gauss-Bonnet theorem and the estimate in part b) of Lemma 1, H. Bray obtained the following result:

Lemma $2([3])$. Assume that $\left(M^{3}, g\right)$ has positive Ricci curvature. Then

$$
I^{\prime \prime}(V) \leq I_{V}^{\prime \prime}(V)=-\frac{\int_{\Sigma}\left|\mathrm{A}_{\Sigma}\right|^{2}+\operatorname{Ric}_{M}(\vec{\nu}, \vec{\nu})}{I(V)^{2}} \leq \frac{4 \pi}{I(V)^{2}}-\frac{3 I^{\prime+}(V)^{2}}{4 I(V)}-\frac{\int_{\Sigma} \mathrm{R}_{M}}{2 I(V)^{2}}
$$

for every isoperimetric surface $\Sigma^{2}$ corresponding to the volume $V$.

The monotonicity of the adapted Hawking mass $m_{H}$ in [3] is crucial:

Lemma 3 ([3]). Let $\left(M^{3}, g\right)$ be a closed Riemannian 3-manifold with $\mathrm{R}_{M} \geq 6$. Then, as a distribution, $m_{H}^{\prime} \geq 0$ on any connected subinterval of $\left.\left(0, \operatorname{vol}\left(M^{3}\right) / 2\right)\right)$ on which (a) every volume is realized by some isoperimetric region with connected boundary, and (b) the isoperimetric profile $I$ is nondecreasing. In particular, if $\operatorname{Ric}_{M}>0$, then $m_{H}$ is nondecreasing on the interval $\left(0, \frac{1}{2} \operatorname{vol}\left(M^{3}\right)\right)$.

Proof. For $\delta \neq 0$ define the difference quotient operator $\left.\Delta_{\delta}\right|_{V} f=\delta^{-1}(f(V+$ $\delta)-f(V))$. Recall that $\Delta_{\delta}$ obeys a product rule and has formal adjoint $-\Delta_{-\delta}$. Moreover, if $f \geq g$ in a neighbourhood of a point $V$ with $f(V)=g(V)$, then $\left.\Delta_{-\delta}\right|_{V} \Delta_{\delta} f \geq\left.\Delta_{-\delta}\right|_{V} \Delta_{\delta} g$ for $\delta \neq 0$ sufficiently small. 
Let $0 \leq \phi \in C_{c}^{1}(a, b)$ be a non-negative test function compactly supported in an interval $(a, b) \subset\left(0, \operatorname{vol}\left(M^{3}\right)\right)$ as in the hypothesis of the lemma. One computes

$$
\begin{aligned}
-\int \phi^{\prime} m_{H} & =-\lim _{\delta \rightarrow 0} \int\left(\Delta_{\delta} \phi\right) \sqrt{I}\left(16 \pi-4 I-\left(\Delta_{\delta} I\right)^{2} I\right) \\
& =\lim _{\delta \rightarrow 0} \int \phi \Delta_{-\delta}\left(\sqrt{I}\left(16 \pi-4 I-\left(\Delta_{\delta} I\right)^{2} I\right)\right) \\
& =\int 2 \phi I^{\prime} I^{\frac{3}{2}}\left(\frac{4 \pi}{I^{2}}-\frac{3 I^{\prime 2}}{4 I}-\frac{3}{I}\right)+\lim _{\delta \rightarrow 0} \int 2 \phi I^{\prime} I^{\frac{3}{2}}\left(-\Delta_{-\delta}\left(\Delta_{\delta} I\right)\right) \\
& \geq \int 2 \phi I^{\prime} I^{\frac{3}{2}}\left(\frac{4 \pi}{I^{2}}-\frac{3 I^{\prime 2}}{4 I}-\frac{3}{I}-\limsup _{\delta \rightarrow 0} \Delta_{-\delta}\left(\Delta_{\delta} I\right)\right) .
\end{aligned}
$$

Here, we have used that $I^{\prime+}(V)=I^{\prime-}(V)$, except possibly at countably many values of $V$, and the fact that $\Delta_{-\delta}\left(\Delta_{\delta} I\right) \leq 0$, which is just the concavity of $I$. By Lemma 2

$$
\begin{aligned}
\left.\limsup _{\delta \rightarrow 0} \Delta_{-\delta}\right|_{V}\left(\Delta_{\delta} I\right) \leq I_{V}^{\prime \prime}(V) & \leq \frac{4 \pi}{I^{2}(V)}-\frac{3 I^{\prime+}(V)^{2}}{4 I(V)}-\frac{\int_{\Sigma_{V}} \mathrm{R}_{M}}{2 I(V)^{2}} \\
& \leq \frac{4 \pi}{I^{2}(V)}-\frac{3 I^{\prime+}(V)^{2}}{4 I(V)}-\frac{3}{I(V)}
\end{aligned}
$$

for almost every $V$ since again $I^{\prime+}(V)=I^{\prime-}(V)$ for all but countably many values of $V$. Together with the hypothesis that $I^{\prime+} \geq 0$, this implies that $-\int \phi^{\prime} m_{H} \geq 0$.

By the concavity of $I$ when $\operatorname{Ric}_{M}>0$ and its symmetry with respect to $\frac{1}{2} \operatorname{vol}\left(M^{3}\right)$, $I_{M}$ is increasing on $\left(0, \operatorname{vol}\left(M^{3}\right) / 2\right)$; hence, by the above, so is $m_{H}$.

The following corollary characterizes equality in Lemma 3 Its proof is implicit in the proof of the scalar curvature rigidity theorem in 3 .

Corollary 1 ([3]). Let $\left(M^{3}, g\right)$ have $\mathrm{R}_{M} \geq 6$ and $\operatorname{Ric}_{M}>0$. If a volume $V_{0} \in$ $\left.\left(0, \operatorname{vol}\left(M^{3}\right) / 2\right)\right]$ is such that $m_{H}\left(V_{0}\right) \leq 0$, then the isoperimetric profile of $M^{3}$ coincides with that of $\mathbb{S}^{3}$ on $\left[0, V_{0}\right]$.

Proof. Lemma 3 implies that $m_{H}^{\prime} \geq 0$ as a distribution on $\left(0, \operatorname{vol}\left(M^{3}\right) / 2\right)$ and hence is a non-decreasing function at least where $m_{H}$ is continuous. Since $I$ is concave, $I^{\prime+}$ can only jump down, and it follows that $m_{H}$ is in fact non-decreasing on all of $\left(0, \operatorname{vol}\left(M^{3}\right) / 2\right]$. Moreover, $\lim _{V \rightarrow 0} m_{H}(V)=0$ (one can use the result of Christodoulou and Yau in the introduction to argue this); and hence if $m_{H}\left(V_{0}\right)=0$, then $m_{H}$ has to vanish identically on $\left(0, V_{0}\right]$. This means that $16 \pi-4 I(V)-$ $I(V) I^{\prime+}(V)^{2} \equiv 0$ on this interval. From the continuity of $I$ it follows that $I^{\prime} \equiv I^{\prime+}$ is continuous and that $I$ is a classical solution of the ODE

$$
I^{\prime}=\sqrt{\frac{16 \pi-4 I}{I}}
$$

on $\left(0, V_{0}\right]$. Since $\operatorname{Ric}_{M}>0, I$ is strictly concave. It is easy to see now that there exists a unique solution with $I(0)=0$ (introducing the function $r=r(V)$ defined implicitly by $I(V)=4 \pi \sin ^{2}(r)$ helps integrate the separated equation where $I(V)<4 \pi)$. An easy computation shows that the adapted Hawking mass of $\mathbb{S}^{3}$ vanishes identically (indeed, the equator is an isoperimetric surface of area $4 \pi$ ), so that indeed $I(V)=I_{\mathbb{S}^{3}}(V)$ on $\left[0, V_{0}\right]$. 
In the next lemma we recall the Taylor series expansion for the volume of small geodesic balls, found by A. Gray and L. Vanhecke in [7] by integration of the expansion of the volume element in geodesic normal coordinates. For our purposes, the expansion of the volume element to fourth order (see also Lemma 3.4 in Chapter 5 in [15]) is sufficient.

Lemma 4 ([7]). Let $M^{3}$ be a Riemannian 3-manifold, $p \in M$ and $0 \leq r \ll 1$. Then

$$
\begin{aligned}
& \operatorname{vol}(B(p, r))= \\
& \frac{4 \pi r^{3}}{3}(\underbrace{1-\frac{\mathrm{R}(p)}{30}}_{:=c_{1}(p)} r^{2}+\underbrace{\frac{1}{6300}\left(4 \mathrm{R}(p)^{2}-2|\operatorname{Ric}(p)|^{2}-9(\Delta \mathrm{R})(p)\right)}_{:=c_{2}(p)} r^{4}+O\left(r^{6}\right)) .
\end{aligned}
$$

In Section 8 of 7], the authors concluded directly from this expansion that a 3-manifold $M^{3}$ has constant sectional curvature (or, equivalently, is Einstein) provided that all small geodesic balls in $M^{3}$ have the same volume as the geodesic balls of the same radius in some fixed simply-connected 3-manifold of constant sectional curvature. In our proof of Theorem 1 we will use the isoperimetric profile $I_{M}$ as a lower bound for the isoperimetric ratio of small geodesic balls, whose Taylor expansion we give in the lemma below. It turns out that this one-sided comparison (along with the lower bound for the scalar curvature) already implies that $M^{3}$ is round, and the proof of this fact given below depends delicately on the sign of the coefficients in the Taylor expansion of the preceding lemma.

Lemma 5. Given $p \in M^{3}$ and $0<V \ll 1$ there exists a unique $r>0$ with $\operatorname{vol}(B(p, r))=V$, and the dependence is smooth. Introducing the variable $W=$ $\left(\frac{3 V}{4 \pi}\right)^{\frac{1}{3}}$ we obtain for the area of the corresponding geodesic sphere the expansion

$$
\operatorname{area}(\partial B(p, r))=4 \pi W^{2}\left(1+c_{1} W^{2}+\left(-\frac{11}{9} c_{1}^{2}+\frac{5}{3} c_{2}\right) W^{4}+O\left(W^{6}\right)\right),
$$

where $c_{1}=c_{1}(p), c_{2}=c_{2}(p)$ are as in the previous lemma.

Proof. This follows from the previous lemma and an elementary calculation.

Theorem 1. Let $\left(M^{3}, g\right)$ be a closed Riemannian manifold with $\mathrm{R}_{M} \geq 6$ and $\operatorname{Ric}_{M}>0$. Then all isoperimetric surfaces of $M^{3}$ have area strictly less than $4 \pi$ unless $\left(M^{3}, g\right)$ is isometric to $\mathbb{S}^{3}$.

Proof. Assume that $I\left(V_{0}\right) \geq 4 \pi$ for some $V_{0} \in\left(0, \operatorname{vol}\left(M^{3}\right) / 2\right]$. Then $m_{H}\left(V_{0}\right) \leq 0$ and Corollary 1 immediately implies that $\operatorname{vol}\left(M^{3}\right)=\operatorname{vol}\left(\mathbb{S}^{3}\right)$ and that $I_{M}=I_{\mathbb{S}^{3}}$.

As a first step, we argue that $\mathrm{R}_{M} \equiv 6$. Fix a point $p \in M^{3}$. For any (small) value of $V>0$ the boundary of the geodesic ball $B(p, r)$ of volume $V$ has surface area $\geq I_{M}(V)=I_{\mathbb{S}^{3}}(V)$. Using that the isoperimetric surfaces of the sphere are just geodesic spheres, we obtain from the previous lemma that

$$
4 \pi W^{2}\left(1-\frac{\mathrm{R}_{M}(p)}{30} W^{2}+O\left(W^{4}\right)\right) \geq 4 \pi W^{2}\left(1-\frac{1}{5} W^{2}+O\left(W^{4}\right)\right)
$$

where again $W=\left(\frac{3 V}{4 \pi}\right)^{\frac{1}{3}}$. Since this inequality holds for all $W>0$ sufficiently small, we conclude that $\mathrm{R}_{M}(p) \leq 6$ and hence, since $p \in M^{3}$ was arbitrary, that $\mathrm{R}_{M} \equiv 6$. 
We now proceed to show that in fact $\operatorname{Ric}_{M} \equiv 2 g$. To see this, we focus on the sixth order term in the above expansion:

$$
\begin{aligned}
& 4 \pi W^{2}\left(1-\frac{1}{5} W^{2}+\left(-\frac{\left|\operatorname{Ric}_{M}(p)\right|^{2}}{1890}-\frac{17}{1575}\right) W^{4}+O\left(W^{6}\right)\right) \\
\geq & 4 \pi W^{2}\left(1-\frac{1}{5} W^{2}+\left(-\frac{\left|\operatorname{Ric}_{\mathbb{S}^{3}}(N)\right|^{2}}{1890}-\frac{17}{1575}\right) W^{4}+O\left(W^{6}\right)\right) .
\end{aligned}
$$

Hence $\left|\operatorname{Ric}_{M}(p)\right|^{2} \leq\left|\operatorname{Ric}_{\mathbb{S}^{3}}(N)\right|^{2}=12$. Since $\mathrm{R}_{M} \equiv 6$ this means that $\operatorname{Ric}_{M} \equiv 2 g$.

It follows that $M^{3}$ is round. Using now that $\operatorname{vol}\left(M^{3}\right)=\operatorname{vol}\left(\mathbb{S}^{3}\right)$ we see that $M^{3}$ must actually coincide with the standard $\mathbb{S}^{3}$.

\section{Min-Oo's COnjecture}

As an application of Theorem 1 we answer in the affirmative the following special case of Min-Oo's conjecture:

Theorem 2. Let $\left(M^{3}, g\right)$ be a compact orientable Riemannian 3-manifold with scalar curvature $\mathrm{R}_{M} \geq 6$, Ricci curvature $\operatorname{Ric}_{M}>0$ and totally geodesic boundary $\partial M^{3}$. If $\operatorname{area}\left(\partial M^{3}\right) \geq 4 \pi$ and $\partial M^{3}$ is an isoperimetric surface of the doubled manifold $\left(\hat{M}^{3}, \hat{g}\right)$, then $\left(M^{3}, g\right)$ is isometric to the upper hemisphere $\mathbb{S}_{+}^{3}$.

Proof. Observe that since we assume that $\partial M^{3}$ is totally geodesic in $M^{3}$, the doubled manifold with the reflected metric is only $\mathcal{C}^{1,1}$ across $\partial M^{3}$. This degree of regularity is enough for the proof of Lemma 3 to pertain. The full assertion now follows from applying the method of Theorem 1 away from the gluing region.

\section{ACKNOWLEDGEMENTS}

This paper forms part of my thesis at Stanford University. I would like to sincerely thank my advisor, Richard Schoen, for his constant support and encouragement and for suggesting this problem to me. I am very grateful to Hubert Bray for his interest in this work and for many stimulating and fun discussions.

\section{REFERENCES}

[1] L. Andersson, M. Cai, G. Galloway: "Rigidity and positivity of mass for asymptotically hyperbolic manifolds," Ann. Henri Poincaré 9 (2008), 1-33. MR2389888

[2] C. Bavard, P. Pansu: "Sur le volume minimal de $\mathbb{R}^{2}$," Ann. Sci. École Norm. Sup. (4) 19 (1986), 479-490. MR0875084 (88b:53048)

[3] H. L. Bray: "The Penrose inequality in general relativity and volume comparison theorems involving scalar curvature," thesis, Stanford University (1997). arXiv:0902.3241

[4] D. Christodoulou, S.-T. Yau: "Some remarks on the quasi-local mass," Contemporary Mathematics, Volume 71, Amer. Math. Soc. (1988), 9-14. MR0954405 (89k:83050)

[5] P. Chrusciel, M. Herzlich: "The mass of asymptotically hyperbolic Riemannian manifolds," Pacific J. Math. 212 (2003), 231-264. MR2038048 (2005d:53052)

[6] J. Corvino: "Scalar curvature deformation and a gluing construction for the Einstein constraint equations," Comm. Math. Phys. 214 (2000), 137-189. MR1794269 (2002b:53050)

[7] A. Gray, L. Vanhecke: "Riemannian geometry as determined by the volumes of small geodesic balls," Acta. Math. 142 (1979), 157-198. MR0521460 (81i:53038)

[8] F. Hang, X. Wang: "Rigidity and non-rigidity results on the sphere," Comm. Anal. Geom. 14 (2006), 91-106. MR2230571 (2007d:53065)

[9] F. Hang, X. Wang: "A rigidity theorem for the hemisphere," preprint (2007). arXiv:0711.4595v2

[10] G. Huisken: "An isoperimetric concept for mass and quasilocal mass," Oberwolfach Rep. 3, No. 1 (2006), 87-88. 
[11] P. Miao: "Positive mass theorem on manifolds admitting corners along a hypersurface," Adv. Theor. Math. Phys. 6 (2002), 1163-1182. MR1982695(2005a:53065)

[12] M. Min-Oo: "Scalar curvature rigidity of asymptotically hyperbolic spin manifolds," Math. Ann. 285 (1989), 527-539. MR.1027758 (91b:53047)

[13] M. Min-Oo: "Scalar curvature rigidity of certain symmetric spaces," CRM Proceedings and Lecture Notes, Volume 15, Amer. Math. Soc. (1998), 127-136. MR1619128 (99e:53056)

[14] A. Ros: "The isoperimetric problem," lecture notes for the Clay Mathematics Institute Summer School on the Global Theory of Minimal Surfaces, Clay Math. Proc., Volume 2, Amer. Math. Soc. (2005), 175-209. MR2167260(2006e:53023)

[15] R. Schoen, S.-T. Yau: "Lectures on Differential Geometry," Conference Proceedings and Lecture Notes in Geometry and Topology, Volume 1, International Press (1994). MR.1333601 (97d:53001)

[16] R. Schoen, S.-T. Yau: "Positivity of the total mass of a general space-time," Phys. Rev. Lett. 43 (1979), 1457-1459. MR0547753 (81c:58024)

[17] Y. Shi, L.-F. Tam: "Positive mass theorem and the boundary behaviors of compact manifolds with nonnegative scalar curvature," J. Differential Geom. 62 (2002), 79-125. MR1987378 (2005b:53046)

[18] X. Wang: "The mass of asymptotically hyperbolic manifolds," J. Differential Geom. 57 (2001), 273-299. MR.1879228 (2003c:53044)

[19] E. Witten: "A new proof of the positive energy theorem," Comm. Math. Phys. 80 (1981), 381-402. MR0626707 (83e:83035)

Department of Mathematics, Massachusetts Institute of Technology, 77 MassaChusetts Avenue, Cambridge, Massachusetts 02139-4307

E-mail address: eichmair@math.mit.edu 